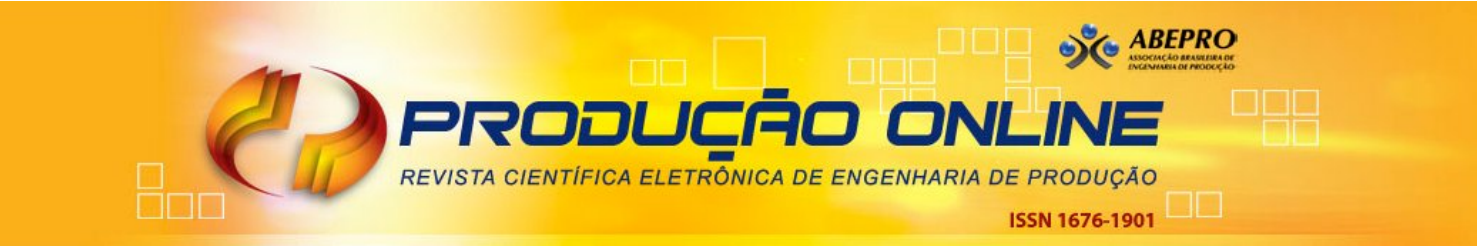

\title{
IMPORTÂNCIA E FERRAMENTAS DE AVALIAÇÃO DE DESEMPENHO PARA REDES DE PEQUENAS E MÉDIAS EMPRESAS: ANÁLISE CRÍTICA DA LITERATURA NACIONAL VERSUS INTERNACIONAL
}

\section{IMPORTANCE AND PERFORMANCE EVALUATION TOOLS FOR SMALL AND MEDIUM COMPANIES: CRITICAL ANALYSIS OF NATIONAL VERSUS INTERNATIONAL LITERATURE}

\author{
Sandro César Bortoluzzi` E-mail: sandro@utfpr.edu.br \\ Sandra Rolim Ensslin** E-mail: sensslin@gmail.com

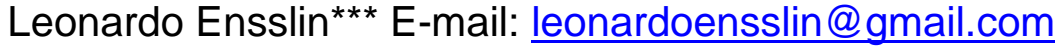 \\ *Universidade Tecnológica Federal do Paraná (UTFPR), Pato Branco, PR \\ **Universidade Federal de Santa Catarina (UFSC), Florianópolis, SC \\ ***Universidade do Sul de Santa Catarina (UNISUL), Florianópolis, SC
}

\begin{abstract}
Resumo: A pesquisa objetiva mapear a importância e as ferramentas de avaliação de desempenho para redes de pequenas e médias empresas. O presente estudo descritivo e qualitativo analisou 33 artigos nacionais e 21 artigos internacionais. Em relação à importância da avaliação de desempenho para redes de pequenas e médias empresas, a literatura destaca: (i) aumenta o sucesso da rede; (ii) é instrumento útil para a gestão; (iii) reforça a competitividade; (iv) consolida a cooperação; e, (v) aumenta a confiança entre os parceiros. Na comparação da literatura nacional versus internacional sobre a importância da avaliação de desempenho para redes de pequenas e médias empresas, percebem-se aspectos similares e complementares, ou seja, não se verifica divergência entre os autores. Os autores utilizam ferramentas consolidadas na literatura, tais como: Balanced Scorecard; Benchmarking; Performance Prism e ferramentas propostas especificamente para avaliar redes de pequenas e médias empresas. As principais dimensões avaliadas são: (i) intercâmbio de informações; (ii) gerenciamento de valor em redes; (iii) nível de maturidade das redes; (iv) benefícios da colaboração; (v) capital social; (vi) eficiência coletiva; (vii) ciclo de vida da rede; (viii) eficiência e ineficiência das redes; e, (ix) existência e intensidade do relacionamento entre os parceiros. A análise crítica em relação ao conceito de avaliação de desempenho adotado no presente estudo permite concluir que as ferramentas propostas ou aplicadas para avaliar as redes de pequenas e médias empresas apresentam lacunas no processo para identificar os critérios, mensurar ordinal e cardinalmente, integrar e gerar ações de aperfeiçoamento.
\end{abstract}

Palavras-chave: Avaliação de Desempenho. Redes de Empresas. Ferramentas de Avaliação de Desempenho. Pequenas e Médias Empresas.

Abstract: The research aims to map the importance and performance evaluation tools for small and medium companies. This descriptive and qualitative study analyzed 33 national articles and 21 international ones. Regarding the importance of performance evaluation for small and medium companies, the literature highlights: (i) it increases the success of the network; (ii) it is useful for management; (iii) it strengthens competitiveness; (iv) it consolidates cooperation; and, (v) it increases trust among partners. Comparing the national versus international literature on the importance of performance evaluation for small and medium companies, it can be noticed similar and complementary aspects, that is, there is not disagreement between the authors. The authors use tools

Revista Produção Online, Florianópolis, SC, v.15, n. 4, p. 1148-1173, out./dez. 2015. 
consolidated in the literature, such as Balanced Scorecard; Benchmarking; Performance Prism and tools proposed specifically to evaluate small and medium networks. The main dimensions evaluated are: (i) exchange of information; (ii) value management in networks; (lii) level of network maturity; (iv) benefits of collaboration; (v) social capital; (vi) collective efficiency; (vii) network life cycle; (viii) efficiency and inefficiency of the networks; and, (ix) existence and intensity of the relationship between partners. The critical analysis regarding the performance evaluation concept adopted in the present study shows that the tools proposed or implemented to evaluate small and medium business networks have gaps in the process to identify criteria, measure ordinal and cardinally, integrate and generate actions of improvement.

Keywords: Performance Evaluation. Corporate Network. Performance Evaluation Tools. Small and Medium Companies.

\section{INTRODUÇÃo}

As tentativas de oferecer um quadro de avaliação de desempenho (AD) para redes de empresas ainda são poucas. No entanto, observa-se a importância dessas ferramentas para a gestão das redes de Pequenas e Médias Empresas (PMEs) (PANICCIA, 1999; ANTONELLI e CAROLEO, 2011).

Os conceitos e as técnicas de medição de desempenho podem apoiar na gestão de eficiência coletiva e melhoria do desempenho das empresas do cluster. Um sistema de medição de desempenho com métricas enfocando os fatores chave de desempenho de um cluster industrial podem ajudar a gerenciar o desempenho de modo a reforçar o posição competitiva de um cluster. A adoção dos conceitos e das práticas de medição de desempenho realiza ações conjuntas entre empresas de um cluster e pode ajudar a consolidar a cooperação, a articulação e a troca de informações entre empresas, bem como desenvolver uma cultura de inovação contínua, contribuindo assim para o desenvolvimento da eficiência coletiva do cluster. Portanto, isso dá origem à necessidade de gerir a eficiência coletiva de um cluster enfocando os conceitos e a prática da gestão de desempenho (CARPINETTI, GALDÁMEZ e GEROLAMO, 2008).

Percebe-se que a literatura apresenta argumentos que indicam que as ferramentas de AD são importantes para o gerenciamento de redes de PMEs. No entanto, essa mesma literatura argumenta que ainda a área de estudo necessita de novas pesquisas e da construção de ferramentas de $A D$ que atenda às necessidades particulares das redes de PMEs.

Dessa forma, o presente estudo pretende contribuir para consolidar um pouco

Revista Produção Online, Florianópolis, SC, v.15, n. 4, p. 1148-1173, out./dez. 2015. 
mais a área de pesquisa e, para isso, busca responder à seguinte pergunta de pesquisa: Qual a importância e as ferramentas da avaliação de desempenho para redes de PMEs? Para responder à pergunta de pesquisa tem-se como objetivo geral mapear, na literatura nacional e na internacional, a importância e as ferramentas de avaliação de desempenho para redes de PMEs. Para alcançar o objetivo geral, estabeleceram-se os seguintes objetivos específicos: (i) extrair dos artigos publicados em periódicos nacionais e internacionais a visão dos diferentes autores sobre a importância da avaliação de desempenho para redes de PMEs; (ii) mapear as ferramentas de avaliação de desempenho propostas ou aplicadas para avaliar o desempenho de redes de pequenas e médias empresas; (iii) cotejar a literatura nacional versus internacional; e, (iv) analisar criticamente as ferramentas de avaliação de desempenho propostas ou aplicadas para avaliar o desempenho de redes de pequenas e médias empresas em função da filiação teórica adotada no presente estudo.

A pesquisa justifica-se pela importância e originalidade (CASTRO, 1977). A importância é em função da contribuição teórica do artigo ao tema $A D$ de redes de Pequenas e Médias Empresas (PMEs). Adicionalmente, a pesquisa contribui com as redes de PMEs existentes no Brasil, pois consolida, em um único trabalho, a importância e as ferramentas de avaliação de desempenho de redes de pequenas e médias empresas, contribuindo com o esclarecimento aos gestores das redes sobre a importância da $A D$ e as principais ferramentas de $A D$ discutidas na literatura nacional e na internacional.

O artigo caracteriza-se como original, pois não se encontrou na literatura publicações com as características aqui apresentadas. Os trabalhos existentes na literatura buscam discutir normalmente uma única ferramenta de AD ou discutem a teoria de redes de empresas com base em trabalhos seminais. Contudo, o presente estudo busca, por meio de um processo estruturado, abranger publicações nacionais e internacionais e essa abrangência possibilita um estudo mais aprofundado sobre a importância e as ferramentas de avaliação de desempenho em redes de pequenas e médias empresas. 
As seguintes delimitações são apresentadas para a construção do presente estudo: (i) foram pesquisados apenas os periódicos presentes no portal de periódicos da Coordenação de Aperfeiçoamento de Pessoal de Nível Superior (CAPES) na data da pesquisa; (ii) o processo de busca para a seleção dos artigos para formar o portfólio bibliográfico é subjetivo em algumas etapas; e, (iii) as bases de dados do portal de periódicos foram selecionadas pelos critérios estabelecidos pelos autores do presente estudo.

\section{REFERENCIAL TEÓRICO}

Este trabalho tem como foco as redes horizontais de PMEs. As redes horizontais são consideradas relações complexas por envolver simultaneamente a cooperação e a competição, onde as empresas concorrentes optam por cooperar, ao mesmo tempo em que são concorrentes. Dessa forma, as relações são favorecidas quando a cooperação concentra esforços para alcançar objetivos comuns, sem tirar a liberdade de ação estratégica e o poder de decisão de seus membros (NEVES et al., 2011). Esses novos modelos organizacionais de cooperação não implicam necessariamente o fim da competição. Cooperação e competição podem conviver lado a lado, e, acima de tudo, há limites cada vez menores entre o fim de uma organização e o início de outra (NEUMANN, HEXSEL e BALESTRIN, 2011).

No entanto, o tema redes de PMEs apresenta, em termos de pesquisas, diversas lacunas a serem preenchidas (NEVES et al., 2011). Uma importante lacuna nas pesquisas de redes de empresas é a consolidação de ferramentas de avaliação de desempenho (PANICCIA, 1999).

Encontram-se na literatura propostas de instrumentos de $A D$ para redes de cooperação, como, por exemplo, os trabalhos de Bititci et al., (2004); Carpinetti, Gerolamo e Galdámez (2007); Adam, Oliveira e Schmidt (2008); Jahn, (2009); dentre outros.

No entanto, as ferramentas propostas na literatura apresentam oportunidades de melhoria, pois a maioria dos trabalhos busca adaptar os modelos desenvolvidos 
para empresas individuais e aplicar tais modelos em redes de cooperação (LIMA, GUERRINI e CARPINETTI, 2011).

Adicionalmente, percebe-se que as ferramentas desenvolvidas para avaliar o desempenho têm foco nos aspectos econômico-financeiros da rede de empresas, sendo que a literatura preconiza que se devem levar em conta medidas financeiras e não financeiras, aspectos qualitativos e quantitativos, aspectos objetivos e subjetivos e que as medidas devem derivar das estratégias da rede (WEGNER e MISOCSKY, 2010).

Pelo exposto, desenvolver ferramentas para avaliar redes de empresas é uma tarefa que exige o atendimento de diversos elementos. No entanto, argumenta-se na literatura que a AD dessas organizações é um passo importante para a consolidação da rede, para trazer transparência ao processo decisório, para aumentar a confiança entre os parceiros, reforçar a competitividade e aumentar a eficiência coletiva (CARPINETTI, GEROLAMO e GALDÁMEZ, 2007; CARPINETTI, GALDÁMEZ e GEROLAMO, 2008).

Dessa forma, após exposta a importância da avaliação de desempenho de redes de empresas, faz-se necessário a filiação teórica dos autores sobre o que é $A D$ organizacional, para que se possam analisar criticamente as ferramentas encontradas na literatura à luz desse conceito. Os autores filiam-se ao seguinte conceito de avaliação de desempenho: é o processo de gestão utilizado para construir, fixar e disseminar conhecimentos por meio da identificação, organização, mensuração e integração dos aspectos necessários e suficientes para medir e gerenciar o desempenho dos objetivos estratégicos de um determinado contexto da organização (ENSSLIN et al., 2010; BORTOLUZZI et al., 2010; BORTOLUZZI, ENSSLIN e ENSSLIN, 2013).

Sendo assim, os autores do presente artigo entendem que a construção de modelos de $A D$ para redes de PMEs deve considerar os seguintes paradigmas: (i) ser construído em forma personalizada para representar os valores e preferências de seus gestores associados às especificidades do ambiente que se propõe avaliar; (ii) reconhecer que os gestores, mesmo vivenciando o contexto diariamente, não têm claro seus objetivos, perguntar a eles quais dos seus objetivos para o contexto irá 
gerar respostas desalinhadas e que os próprios gestores posteriormente questionarão; (iii) o processo utilizado deve reconhecer essas limitações de conhecimento e ajudar aos gestores a construir seu entendimento segundo seus valores e preferências; (iv) os objetivos contidos no modelo de AD são fruto não só dos valores e preferências individuais do gestor, mas também de como este é influenciado pelo contexto onde opera; (v) a construção do modelo de AD requer a participação contínua do gestor para assegurar a construção de seu conhecimento sobre o problema e ter legitimidade; (vi) para dar fundamentação e validade às mensurações realizadas, estas necessitam atender aos requisitos da Teoria da Mensuração; e, (vii) o modelo deve, em cada uma de suas etapas de construção e uso, ter o reconhecimento do gestor quando da legitimidade do conhecimento construído e sua representação no modelo explícito, assim como garantir que todos os instrumentos utilizados tenham reconhecimento científico (ENSSLIN et al., 2010).

Nesse contexto, entende-se que os modelos de AD a serem desenvolvido para redes de PMEs devem levar em consideração suas particularidade, ou seja, sua cultura organizacional, a percepção de seus gestores, o contexto regional em que atua, os demais atores envolvidos com a gestão da rede. Resumindo, deve-se construir um modelo que atenda às características da rede a ser avaliada, e não modelos genéricos que possam ser aplicados a qualquer contexto.

\section{METODOLOGIA DA PESQUISA}

\subsection{Enquadramento metodológico}

O enquadramento metodológico da pesquisa é apresentado na Figura 1. 
Figura 1 - Enquadramento metodológico da pesquisa

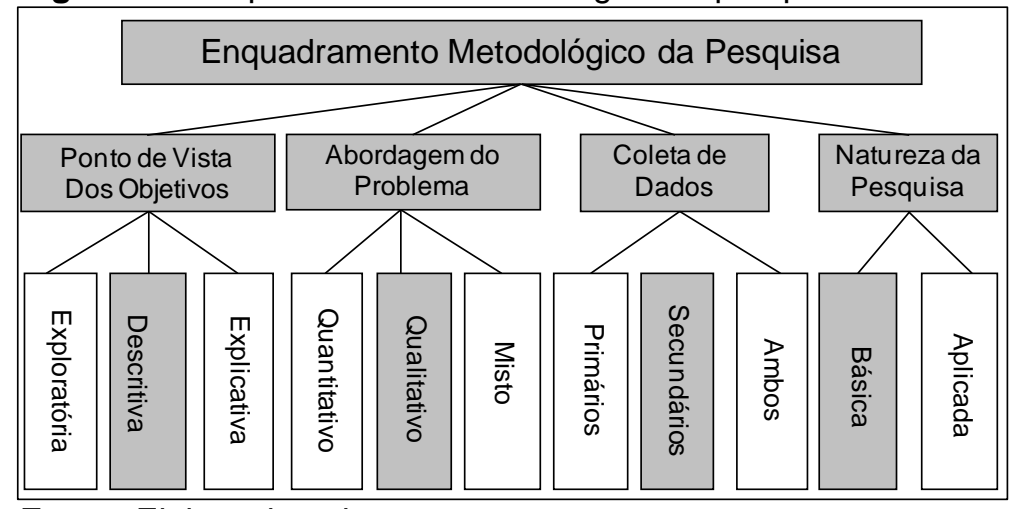

Fonte: Elaborado pelos autores

A pesquisa caracteriza-se como descritiva, pois busca consolidar, em um único estudo, as características de uma amostra de publicações científicas sobre a importância e as ferramentas de avaliação de desempenho (AD) de redes de empresas e também por analisar criticamente essas informações à luz da filiação teórica de AD adotado pelos autores (GIL, 1999). Em relação à abordagem do problema, a pesquisa é qualitativa, pois busca responder à pergunta de pesquisa por meio do mapeamento das informações apresentadas nos artigos científicos e por não usar instrumentos estatísticos para analisar essas informações (RICHARDSON, 2008).

A coleta de dados foi realizada em artigos publicados em periódicos nacionais e internacionais, o que caracteriza a pesquisa como coleta de dados secundária (RICHARDSON, 2008). A natureza da pesquisa é básica, pois se restringe às discussões teóricas sobre o tema Redes de Empresas, ou seja, não tem como foco a aplicação do conhecimento gerado em redes de empresas (GIL, 1999).

\subsection{Procedimentos para busca bibliográfica}

Para formar o portfólio bibliográfico nacional e o internacional, utilizou-se o processo Knowledge Development Process-Constructivist (ProKnow-C), utilizado em várias pesquisas científicas (ROSA et al., 2011; LACERDA, ENSSLIN e ENSSLIN, 2012; ENSSLIN et al., 2014; DUTRA et al., 2015), dentre outras.

Em relação à busca em periódicos internacionais, o processo divide-se em duas principais fases: (i) seleção do banco de artigos bruto; e, (ii) filtragem do banco de artigos. 
A primeira fase é subdividida, por sua vez, em três etapas, quais sejam: (i) definir palavras-chave; (ii) definir bases de dados; e, (iii) buscar artigos nas bases de dados.

Definiram-se os eixos de pesquisa, salientados nos retângulos na cor cinza, na Figura 2 e, na sequência, as palavras-chave apresentadas nos colchetes na Figura 2.

Figura 2 - Eixos de pesquisas e palavras-chave de AD em redes de PMEs

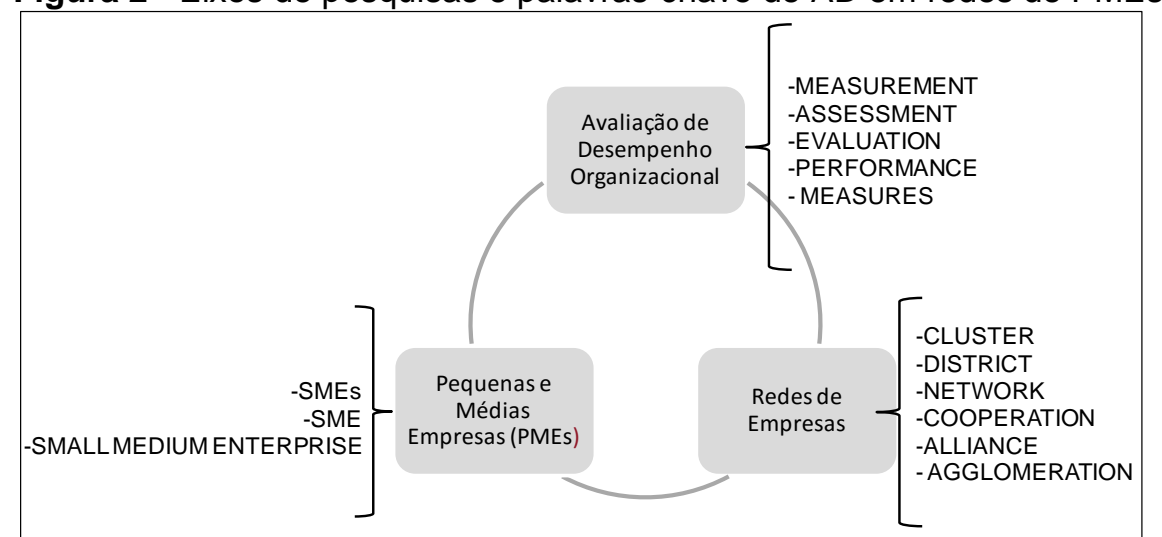

Fonte: Elaborado pelos autores

Com base na definição das palavras-chave, todas as possíveis combinações entre as palavras-chave dos eixos de pesquisa foram formadas como, por exemplo, SMES AND measurement AND cluster. Cabe salientar que a busca foi realizada por meio das 90 possíveis combinações entre as palavras-chave com o uso da expressão booliana AND que indica que o retorno de artigos acontece quando as três palavras-chave aparecem nos campos de busca.

No desenvolvimento da segunda etapa, inicialmente definiram-se as bases de dados para a realização da pesquisa. Como critérios para a seleção das bases de dados foram estabelecidos os seguintes aspectos: (i) as bases de dados deveriam constar no portal de periódicos da Coordenação de Aperfeiçoamento de Pessoal de Nível Superior (CAPES); (ii) para ser escolhida, a base de dados deveria estar em uma das seguintes três grandes áreas da CAPES: Ciências Sociais Aplicadas, Engenharias e Multidisciplinar, pois essas áreas são as mais alinhadas com o tema ilustrado na pesquisa; (iii) as bases de dados deveriam disponibilizar ferramenta de busca com a possibilidade de uso de expressões booleanas; e (iv) as ferramentas de busca das bases de dados deveriam disponibilizar a possibilidade de pesquisar 
nos campos: título, resumo e palavras-chave. Em função dos critérios, foram identificadas e pesquisadas 23 bases de dados.

A terceira etapa consistiu em realizar a busca em cada base de dados por meio das combinações de palavras-chave. Salienta-se que os campos de busca foram: título, resumo e palavras-chave do artigo. Destaca-se também que não se restringiu a busca em relação ao período de tempo, ou seja, buscou-se o intervalo de datas disponibilizado pelas bases de dados. Esse processo resultou no banco de artigos bruto preliminar de 3.375 artigos.

A segunda fase, filtragem do banco de artigos é subdividida, por sua vez, nestas cinco etapas: (i) eliminação de artigos repetidos; (ii) alinhamento pela leitura do título; (iii) alinhamento quanto ao reconhecimento científico; (iv) alinhamento pela leitura do resumo; e (v) alinhamento pela leitura integral dos artigos.

A primeira etapa da fase de filtragem do banco de artigos refere-se à importação dos artigos para um software de gerenciamento bibliográfico. Dessa forma, importou-se para o software Endnote os 3.375 artigos do banco de artigos bruto. Após a importação, o software permite a exclusão dos artigos repetidos. Assim, foram excluídos 2.289 artigos repetidos, e restaram 1.086 artigos não repetidos.

A etapa seguinte foi a leitura dos 1.086 títulos com o objetivo de verificar 0 alinhamento dos artigos ao tema. Cabe salientar que, nessa etapa, buscou-se excluir os artigos que apresentavam total desalinhamento com o tema; na dúvida o artigo foi mantido para análise mais detalhada nas próximas etapas. Como resultado do processo de leitura dos títulos, restaram 501 artigos alinhados ao tema (foram excluídos 585 artigos que não apresentavam alinhamento).

A próxima etapa consiste na identificação do reconhecimento científico dos artigos. Nesse processo, o reconhecimento científico é aferido pelas citações em outros trabalhos científicos; assim, identificou-se a quantidade de vezes que cada artigo foi citado. Para fins de padronização, buscou-se a quantidade de citações no Google acadêmico. Foram identificados os 215 artigos mais citados (97\% das citações) e os 286 artigos menos citados (3\% das citações). 
A seguir, foi feita a leitura dos 215 resumos dos artigos com maior reconhecimento científico com o objetivo de verificar o alinhamento do artigo com o tema de pesquisa. Adicionalmente aos 215 artigos, foram incorporados 27 artigos das referências do portfólio bibliográfico em função do teste da sua representatividade. Após essa etapa, restaram 57 artigos que foram "guardados" no repositório $A$.

A etapa seguinte teve o objetivo de filtrar os artigos com menor reconhecimento científico, ou seja, os 286 artigos com menor reconhecimento científico foram analisados para identificar aqueles que continuariam no processo. Os 17 artigos identificados nessa etapa foram "guardados" no repositório $B$. Na sequência, juntaram-se os artigos "guardados" nos repositórios $A$ (57 artigos) e $B$ (17 artigos), formando um único repositório, denominado $C$, composto por 74 artigos.

A etapa final da filtragem do banco de artigos consiste em fazer o filtro quanto ao alinhamento do artigo integral. Após a eliminação dos artigos que não estavam com o texto completo disponível e leitura integral dos demais artigos, chegou-se a um portfólio bibliográfico internacional de 21 artigos científicos sobre avaliação de desempenho em redes de pequenas e médias empresas.

Em relação aos periódicos nacionais, a busca ocorreu nos periódicos da área de Administração, Contabilidade e Turismo e Engenharia III, classificados com Estrato Qualis A1 a B5, pela Coordenação de Aperfeiçoamento de Pessoal de Nível Superior (CAPES).

O processo de seleção de artigos relevantes sobre o tema da presente pesquisa teve inicio com a seleção de artigos relacionada à AD organizacional e, na sequência, selecionaram-se os artigos com foco em $A D$ de redes de pequenas e médias empresas.

Dessa forma, a primeira fase do processo buscou selecionar artigos que abordassem AD organizacional. Procurou-se, na primeira etapa, identificar os periódicos nacionais da área de Administração, Contabilidade e Turismo e Engenharia III, classificados com Estrato Qualis/CAPES A1 a B5. Foram pesquisados 408 periódicos científicos das áreas de Administração Ciências Contábeis e Turismo e Engenharias III. 
A segunda etapa do processo consistiu em pesquisar os 408 periódicos por meio da incidência das seguintes palavras-chave no título, no resumo ou nas palavras-chave do artigo: (i) avaliação; (ii) desempenho; (iii) performance; (iv) decisão; e, (v) mensuração.

Após a realização da busca por meio das palavras-chave, retornaram 22.392 artigos científicos que foram cadastrados em um software de gerenciamento bibliográfico.

$\mathrm{Na}$ etapa seguinte, buscou-se o alinhamento do artigo ao tema AD pela leitura dos títulos, sendo que, após a leitura dos 22.392 títulos, foi constatado que apenas 5.591 títulos estavam alinhados, e os demais 16.801 foram eliminados por não alinhamento.

A próxima etapa realizada foi verificar o alinhamento do artigo ao tema AD em função da leitura dos resumos. Após a leitura dos 5.591 resumos, identificou-se que 668 artigos estavam alinhados, e os demais 4.923 artigos foram eliminados por não alinhamento.

A segunda fase do processo consistiu em verificar o alinhamento dos 668 artigos com o tema $A D$ em pequenas e médias empresas. Dessa forma, primeiro realizou-se a leitura dos 668 resumos, sendo identificados 55 artigos alinhados ao tema. Na sequência, foi feita a leitura integral dos 55 artigos para verificar os que estavam totalmente alinhados ao tema AD em redes de PMEs. Após a leitura dos 55 artigos, selecionaram-se 33 artigos alinhados e que formam o Portfólio Bibliográfico nacional.

\subsection{Procedimentos para análise dos dados}

Mapearam-se os artigos com o objetivo de extrair das pesquisas analisadas a importância e as ferramentas de avaliação de desempenho de redes de PMEs. Para realizar o mapeamento dos 21 artigos do portfólio bibliográfico internacional e dos 33 artigos do portfólio bibliográfico nacional, procedeu-se à leitura integral desses artigos e foram extraídas as informações relacionadas à importância e às ferramentas de AD em redes de PMEs. A análise de conteúdo (BARDIN, 2004) foi 
utilizada para ler, interpretar e processar as informações dos artigos científicos selecionados o que permitiu consolidar a importância e as ferramentas de AD em redes de PMEs. Essas informações foram registradas em uma planilha eletrônica, organizadas por um número sequencial de cada trabalho, o nome dos autores, o ano de publicação, o periódico em que o artigo foi publicado, uma coluna com a importância da AD em redes de PMEs e outra coluna com a proposta de ferramenta para avaliar o desempenho de redes de empresas.

\section{APRESENTAÇÃO E DISCUSSÃO DOS RESULTADOS}

\subsection{Importância da Avaliação de desempenho para Redes de Pequenas e Médias Empresas}

O Quadro 1 apresenta argumentos sobre a importância da avaliação de desempenho (AD) para redes de PMEs na perspectiva dos autores que publicaram artigos em periódicos internacionais.

No Quadro 1, percebe-se a importância de um sistema de AD para redes de PMEs. Os autores argumentam a importância de usar medidas de desempenho para gerenciar redes de empresas (ANTONELLI e CAROLEO, 2011; CAMARINHOMATOS e ABREU, 2007). De forma mais ampla do que somente criar um conjunto de indicadores, outras pesquisas argumentam que é necessário utilizar os conceitos e técnicas de gestão e medição de desempenho para gerenciar a eficiência coletiva e a melhorar o desempenho de empresas envolvidas na cooperação (CARPINETTI, GALDÁMEZ e GEROLAMO, 2008). Segundo esses autores, avaliar o desempenho de redes de empresas permite reforçar a posição competitiva das empresas e do cluster, pode ajudar a consolidar a cooperação e a confiança entre os membros da rede, melhora a troca de informações e gera uma massa crítica que auxilia no desenvolvimento de inovações.

Algumas pesquisas também reforçam que a técnica de benchmarking é uma das formas de avaliar o desempenho e melhorar a troca de informações e 
conhecimentos entre os membros da rede e possibilita a troca de experiências das melhores práticas e a comparação de indicadores de desempenho.

Quadro 1 - Importância da AD para redes de PMEs identificadas na literatura internacional Importância da Avaliação de Desempenho para redes de PMEs

Usar medidas de desempenho para gerenciamento é um requisito importante para o sucesso de uma rede de PMEs.

No contexto das redes de empresas, a definição de um conjunto de indicadores de desempenho pode ser um instrumento útil para a gestão de redes colaborativas.

Os conceitos e técnicas de medidas de desempenho podem ser de grande valia na gestão de eficiência coletiva e melhoria do desempenho das empresas do cluster. Um sistema de medidas de desempenho com métricas enfocando os fatores-chave de desempenho de um cluster industrial pode ajudar a gerenciar o desempenho de modo a reforçar a posição competitiva de um cluster como um todo. A adoção dos conceitos e práticas de medidas de desempenho realiza ações conjuntas entre empresas de um cluster e pode ajudar a consolidar a cooperação, articulação e troca de informações entre empresas, bem como desenvolver uma cultura de inovação contínua, contribuindo assim para o desenvolvimento da eficiência coletiva do cluster.

Um banco de dados de benchmarking pode ser um poderoso instrumento para a partilha de informação e promoção de ações conjuntas entre empresas dentro do cluster.

Um processo de avaliação e medidas de desempenho das ações conjuntas é importante, a fim de dar um feedback estratégico para a gestão da rede. Dessa forma, a implementação de um sistema de medidas de desempenho para a gestão da cooperação da rede como um todo é altamente recomendado.

A medidas de desempenho das empresas ligadas em redes ganha mais relevância na prática.

Observa-se a importância das ferramentas de AD para a gestão de redes de PMEs.

Fonte: Elaborado pelos autores

A medição de desempenho das empresas ligadas em rede ganha cada vez mais relevância na prática (JAHN, 2007). No entanto, avaliar o desempenho de redes colaborativas é um desafio que ainda não encontrou uma solução concreta (PANICCIA, 1999). Os autores argumentam que a maioria dos sistemas de AD de redes foca apenas nas empresas individuais, sendo que algumas tentativas têm sido feitas, mas não há consenso ainda sobre como definir medidas adequadas e avaliálas. Entretanto, observa-se a importância das ferramentas de AD para a gestão de redes de empresas (PANICCIA, 1999).

No Quadro 2, estão argumentos sobre a importância da AD para redes de pequenas e médias empresas na perspectiva dos autores que publicaram artigos em periódicos nacionais sobre AD em redes de PMEs. 
Quadro 2 - Importância da Avaliação de Desempenho para redes de Pequenas e Médias Empresas identificadas na literatura nacional

\begin{tabular}{|l|c|}
\hline \multicolumn{1}{|c|}{ Importância da Avaliação de Desempenho de redes de empresas } & Autor(es) \\
\hline $\begin{array}{l}\text { Um modelo de Sistema de Medição de Desempenho (SMD) pode direcionar o } \\
\text { processo de melhoria e mudança das PMEs do Arranjo Produtivo Local (APL), } \\
\text { promover a gestão colaborativa e aprimorar a tomada de decisão ou a }\end{array}$ & $\begin{array}{c}\text { Galdámez, } \\
\text { Carpinetti e } \\
\text { coordenação das ações planejadas pelas instituições, empresas e outros órgãos } \\
\text { que fazem parte dos APLs. É um instrumento que pode ajudar a monitorar o } \\
\text { desempenho das PMEs inseridas em uma rede de cooperação empresarial e } \\
\text { direcionar as iniciativas coletivas ou ações de melhoria para as áreas (recursos } \\
\text { humanos, administração financeira, comercial, produção e mercado) com } \\
\text { resultados insatisfatórios no APL). }\end{array}$ \\
\hline $\begin{array}{l}\text { Para a gestão desses arranjos produtivos e análise de sua competitividade, torna- } \\
\text { se necessária a Avaliação de Desempenho do arranjo como um todo. }\end{array}$ & $\begin{array}{c}\text { Hansen e } \\
\text { Oliveira } \\
\text { (2009) }\end{array}$ \\
\hline $\begin{array}{l}\text { O desafio das redes de cooperação consiste na avaliação dos resultados, sendo } \\
\text { que, para isso, é importante desenvolver ferramentas de AD que considerem o } \\
\text { contexto específico da rede, e não simplesmente das empresas individuais. A AD } \\
\text { é um instrumento relevante para a gestão de redes de empresas. No entanto, tem } \\
\text { recebido pouca atenção da literatura. }\end{array}$ & $\begin{array}{c}\text { Megner e } \\
\text { (2010) }\end{array}$ \\
\hline $\begin{array}{l}\text { Pesquisas no âmbito organizacional e em outras áreas buscam identificar os } \\
\text { fatores que influenciam na performance de redes de cooperação. }\end{array}$ & $\begin{array}{c}\text { Pereira e } \\
\text { Venturini } \\
\text { (2006) }\end{array}$ \\
\hline $\begin{array}{l}\text { A realização da AD por meio do benchmarking assume especial importância, } \\
\text { dado que permite, por um lado, estabelecer padrões de referência nos } \\
\text { indicadores de desempenho e, por outro lado, identificar desvios e propor } \\
\text { medidas corretivas. }\end{array}$ & $\begin{array}{c}\text { Silva et al. } \\
(2008)\end{array}$ \\
\hline $\begin{array}{l}\text { O desenvolvimento de modelos de gestão para aglomerações produtivas, com } \\
\text { estruturas de governança e indicadores de desempenho se traduz, dessa forma, } \\
\text { em interessante linha de pesquisa acadêmica. }\end{array}$ & $\begin{array}{c}\text { Cassanego } \\
\text { Junior e } \\
\text { Maehler } \\
\text { (2010) }\end{array}$ \\
\hline
\end{tabular}

Fonte: Elaborado pelos autores

No Quadro 2, percebe-se a importância de um sistema de AD para redes de pequenas e médias empresas. Os autores afirmam que um Sistema de Medição de Desempenho (SMD) pode direcionar o processo de melhoria e mudança das PMEs do APL (GALDÁMEZ, CARPINETTI e GEROLAMO, 2009). Outros autores argumentam que, para realizar a gestão de um arranjo produtivo, se faz necessário desenvolver um sistema de AD (HANSEN e OLIVEIRA, 2009). Alguns autores também afirmam que a $A D$ de redes é uma interessante linha de pesquisa (CASSANEGO JUNIOR e MAEHLER, 2010).

Em relação à importância da $A D$ em redes de PMEs, a literatura internacional versus a nacional apresenta aspectos similares e complementares, ou seja, não se verifica divergência entre os autores sobre a importância da AD em redes de PMEs, sendo que o aspecto mais presente é que a $A D$, em redes de PMEs, contribui com a 
gestão dessas organizações e que aumenta a possibilidade de sucesso da rede por meio da transparência das informações, aspecto importante para aumentar a confiança entre os parceiros.

\subsection{Ferramentas da Avaliação de Desempenho para redes de PMEs}

No Quadro 3, está a síntese do processo desenvolvido pelas ferramentas de AD para redes de PMEs, identificados nos artigos publicados em periódicos internacionais.

Quadro 3 - Ferramentas de Avaliação de Desempenho para redes de Pequenas e Médias Empresas identificadas na literatura internacional

\begin{tabular}{|c|c|}
\hline Síntese do processo desenvolvido pela ferramenta & Autor(es) \\
\hline $\begin{array}{l}\text { A metodologia objetiva mostrar que é possível identificar os padrões de colaboração } \\
\text { dentro de um cluster, por meio da análise da estrutura de intercâmbio de informaçôes. }\end{array}$ & $\begin{array}{c}\text { Antonelli e } \\
\text { Caroleo (2011) }\end{array}$ \\
\hline $\begin{array}{l}\text { Propõem-se quatro perspectivas para analisar a criação e gerenciamento de valor em } \\
\text { redes: proposição de valor para o acionista, para a empresa individual; intra-rede e } \\
\text { valor da rede. }\end{array}$ & $\begin{array}{l}\text { Bititci et al. } \\
\text { (2004) }\end{array}$ \\
\hline $\begin{array}{l}\text { Propõem um modelo de sinergia com o objetivo de avaliar o nível de maturidade das } \\
\text { redes em quatro perspectivas: sinergia estratégica, operacional, cultural e comercial. }\end{array}$ & $\begin{array}{l}\text { Bititci et al. } \\
\text { (2007) }\end{array}$ \\
\hline $\begin{array}{l}\text { Propõem-se seis níveis: (i) empresas se reunem para cooperar; (ii) papel mais ativo } \\
\text { das empresas individuais; (iii) criação de uma estrutura formal e legal; (iv) na rede, } \\
\text { cada gestor será então capaz de afirmar sua competência; (v) busca de novos } \\
\text { mercados conjuntamente; e (vi) planejar a estruturação e realização dos seus próprios } \\
\text { produtos. }\end{array}$ & $\begin{array}{l}\text { Burlat, } \\
\text { Besombes e } \\
\text { Deslandres } \\
(2003)\end{array}$ \\
\hline $\begin{array}{l}\text { Os autores apresentam uma série de indicadores para avalir os benefícios da } \\
\text { colaboração, tais como: benefícios individuais gerados, taxa de benefícios recebidos e } \\
\text { taxa de benefícios constribuídos. }\end{array}$ & $\begin{array}{l}\text { Camarinha- } \\
\text { Matos e Abreu } \\
(2007)\end{array}$ \\
\hline $\begin{array}{l}\text { Propõem avaliar o desempenho de redes de PMEs por meio de quatro perspectivas: } \\
\text { capital social; resultados econômicos e sociais, desempenho das empresas } \\
\text { individuais e eficiência coletiva. }\end{array}$ & $\begin{array}{l}\text { Carpinetti, } \\
\text { Galdámez } \\
\text { e Gerolamo } \\
(2008)\end{array}$ \\
\hline $\begin{array}{l}\text { Da ferramenta Balanced Scorecard desenvolve-se indicadores em } 4 \text { perspectivas: } \\
\text { capital social; resultado social e econômico; desempenho das empresas individuais e } \\
\text { eficiência coletiva. Da ferramenta Performance Prism identifica-se as necessidades } \\
\text { dos stakeholders e a orientação estratégica, que se transforma em objetivos de } \\
\text { desempenho para mensurar. }\end{array}$ & $\begin{array}{l}\text { Carpinetti, } \\
\text { Gerolamo e } \\
\text { Galdámez } \\
(2007)\end{array}$ \\
\hline $\begin{array}{l}\text { Propõem-se uma ferramenta de Benchmarking pela definição de indicadores e o } \\
\text { desenvolvimento de um site para as empresas compararem os melhores resultados. }\end{array}$ & $\begin{array}{l}\text { Carpinetti e } \\
\text { Oiko (2008) }\end{array}$ \\
\hline $\begin{array}{l}\text { São cinco etapas: identificar os stakeholders; definir os objetivos; implantar os } \\
\text { projetos de melhoria; avaliar o desempenho e desenvolver uma infra-estrutura de } \\
\text { apoio à gestão. }\end{array}$ & $\begin{array}{c}\text { Gerolamo et al. } \\
(2008)\end{array}$ \\
\hline $\begin{array}{l}\text { Identificou-se } 36 \text { indicadores agrupados em } 6 \text { categorias: (i) eficiência; (ii) o perfil do } \\
\text { empregado; (iii) os prazos de entrega; (iv) flexibilidade de gestão; (v) qualidade; e (vi) } \\
\text { inovação. Utiliza-se o benchmarking para comparação das melhores práticas. }\end{array}$ & $\begin{array}{l}\text { Grando e } \\
\text { Belvedere } \\
(2006)\end{array}$ \\
\hline $\begin{array}{l}\text { São } 24 \text { variáveis em cinco fases: análise estratégica e decisão de cooperar; busca de } \\
\text { parceiros; desenvolvimento do projeto de parceria; gestão da parceria; e } \\
\text { encerramento da parceria. }\end{array}$ & $\begin{array}{l}\text { Hoffmann e } \\
\text { Schlosser } \\
(2001)\end{array}$ \\
\hline
\end{tabular}

Revista Produção Online, Florianópolis, SC, v.15, n. 4, p. 1148-1173, out./dez. 2015. 
Quadro 3 - Ferramentas de Avaliação de Desempenho para redes de Pequenas e Médias Empresas identificadas na literatura internacional

\begin{tabular}{|c|c|}
\hline Síntese do processo desenvolvido pela ferramenta & Autor(es) \\
\hline $\begin{array}{l}\text { A fase de preparação é dividida nas etapas: (i) determinação dos parâmetros de } \\
\text { desempenho; (ii) determinação das escalas de mensuração e funções de valor; (iii) } \\
\text { determinação das taxas. A fase de utilização e composta pelas etapas: (i) } \\
\text { mensuração do desempenho; (ii) avaliação do desempenho; e (iii) cálculo do valor } \\
\text { global de desempenho. }\end{array}$ & Jahn (2009) \\
\hline $\begin{array}{l}\text { Duas fases para construção do modelo. Na primeira, os autores apontam que deveria } \\
\text { ser considerada, a proximidade geográfica, a cultura para o empreendedorismo; } \\
\text { massa crítica das empresas e construção de confiança. Na segunda, deveriam ser } \\
\text { consideradas as medidas de competitividade: produtividade, especialização, } \\
\text { inovação, custos e confiança. }\end{array}$ & $\begin{array}{c}\text { Karaev, } \\
\text { Koh e } \\
\text { Szamosi (2007) }\end{array}$ \\
\hline $\begin{array}{l}\text { Propõem-se avaliar redes em três níveis: individual; da empresa e da rede, } \\
\text { considerando fatores de conduta e estruturais. Com isso. analisa-se o desempenho } \\
\text { das ações conjuntas. }\end{array}$ & $\begin{array}{l}\text { Klint e Sjoberg } \\
\quad(2003)\end{array}$ \\
\hline $\begin{array}{l}\text { Os autores propõem medidas de desempenho para as seguintes perspectivas no } \\
\text { interior das fases do ciclo de vida da rede: informações e troca de conhecimentos; } \\
\text { desempenho operacional; resultado financeiro; saúde da colaboração; resultado } \\
\text { econômico e social. }\end{array}$ & $\begin{array}{l}\text { Lima, Guerrini, } \\
\text { Carpinetti } \\
\text { (2011) }\end{array}$ \\
\hline $\begin{array}{l}\text { São sete etapas: identificação territorial das unidades; seleção da amostra; } \\
\text { operacionalização do conceito de identidade; recolha de dados e pesquisas local; } \\
\text { entrevistas e questionários com técnicos qualificados; cálculo de índices; análise de } \\
\text { correlação. }\end{array}$ & Paniccia (1999) \\
\hline $\begin{array}{l}\text { O modelo considera três elementos: eficiência coletiva, dividida em ações conjuntas e } \\
\text { externalidades; estímulo de políticas, dividido em política nacional e instituições e } \\
\text { política local e instituições e integração social, dividida em coesão social e } \\
\text { autorrealização. }\end{array}$ & Parrilli (2009) \\
\hline $\begin{array}{l}\begin{array}{l}\text { Propõe avaliar se a participação em uma rede de empresas aumenta a } \\
\text { competitividade das empresas individuais no longo prazo. }\end{array} \\
\end{array}$ & $\begin{array}{l}\text { Rosenfeld } \\
(1996)\end{array}$ \\
\hline $\begin{array}{l}\text { A ferramenta busca avaliar o desempenho em três dimensões: (i) estrutura } \\
\text { operacional; (ii) arranjo organizacional; e (iii) interações com o ambiente } \\
\text { socioeconômico. A avaliação é realizada em variáveis nas dimensões estabelecidas e } \\
\text { comparada com as melhores práticas. }\end{array}$ & Villa (2007) \\
\hline
\end{tabular}

Fonte: Elaborado pelos autores

Constatam-se, no Quadro 3, iniciativas de ferramentas para avaliar o desempenho de redes de empresas. Essas ferramentas buscam preencher lacunas identificadas na literatura, e cada proposta apresenta particularidades que, na visão de seus autores, conseguem atender, de forma mais adequada, à necessidade das redes de cooperação.

Percebe-se qua várias ferramentas apresentadas no contexto internacional utilizam-se das de Balanced Scorecard, Benchmarking e Performance Prism, e também outras ferramentas são propostas originais para avaliar o desempenho de redes de empresas.

As ferramentas propostas pelos autores buscam avaliar dimensões específicas de redes, tais como: intercâmbio de informações; gerenciamento de 
valor em redes; nível de maturidade das redes; consideração das empresas individuais e a rede como um todo; benefícios da colaboração; capital social; eficiência coletiva; especialização; inovação; confiança; e ciclo de vida da rede.

No Quadro 4, está a síntese do processo desenvolvido pelas ferramentas de $A D$ de redes de empresas, identificado nos artigos publicados em periódicos nacionais.

Constatam-se, no Quadro 4, as propostas de ferramentas de AD em redes de PMEs publicadas em periódicos nacionais, visto que, na visão dos autores, essas ferramentas conseguem atender ao contexto das redes de cooperação. As ferramentas propostas pelos autores buscam avaliar dimensões específicas de redes, tais como: eficiência coletiva, capital social; desempenho das empresas individuais e da rede como um todo; saúde da relação cooperativa; eficiência e ineficiência das redes; existência e intensidade do relacionamento entre os parceiros.

A comparação das ferramentas propostas no artigos publicados em periódicos internacionais versus nacionais permite concluir que existem congruências e divergências entre os diferentes autores. Identificam-se autores que propõem ferramentas que apresentam natureza essencialmente qualitativa, e outros autores, apresentam natureza mais quantitativa. No entanto, nos periódicos nacionais as ferramentas de natureza quantitativa são em maior número em comparação com os artigos publicados em periódicos internacionais.

Quadro 4 - Ferramentas de Avaliação de Desempenho para redes de Pequenas e Médias Empresas identificadas na literatura nacional

\section{Síntese do processo desenvolvido pela ferramenta} (continua)

São cinco dimensões: perspectiva econômica e social; do meio ambiente; do desempenho das PMEs; da eficiência coletiva; e do capital social. O número de indicadores de desempenho é bastante reduzido, conforme necessidade explicitada no referencial teórico.

São nove passos: definição do segmento produtivo e do negócio; mapeamento e análise preliminar da cadeia; identificação do desempenho competitivo do arranjo; desdobramento das medidas de desempenho competitivo do arranjo; desdobramento das medidas de desempenho competitivo da cadeia; estabelecimento de bancos de dados de todas as medidas de desempenho; identificação e análise do elo fraco do arranjo; realinhamento dos processos do elo fraco; e retorno à etapa de identificação do novo elo fraco. 
Quadro 4 - Ferramentas de Avaliação de Desempenho para redes de Pequenas e Médias Empresas identificadas na literatura nacional

\begin{tabular}{|c|c|}
\hline Síntese do processo desenvolvido pela ferramenta & Autor(es) \\
\hline $\begin{array}{l}\text { São três dimensões: contribuição das empresas individuais para a rede; saúde da } \\
\text { relação cooperativa; e resultados obtidos. Os autores buscam agregar ao modelo a } \\
\text { abordagem da produção de sentido que cada empresário faz da sua inserção na } \\
\text { rede. }\end{array}$ & $\begin{array}{l}\text { Wegner e } \\
\text { Misocsky } \\
(2010)\end{array}$ \\
\hline $\begin{array}{l}\text { A ferramenta utilizada pelo autor é a análise envoltória de dados. O objetivo é } \\
\text { construir um conjunto de referência convexo, com base nos próprios dados das } \\
\text { firmas e então classificá-las em eficientes ou ineficientes, tendo como referencial } \\
\text { essa superfície formada. }\end{array}$ & $\begin{array}{l}\text { Oliveira e } \\
\text { Gomes } \\
(2004)\end{array}$ \\
\hline $\begin{array}{l}\text { Os autores realizaram a AD pela escolha de direcionadores de competitividade e } \\
\text { seus respectivos subfatores. Após identificar os direcionadores buscam qualificar a } \\
\text { intensidade do impacto dos subfatores e sua contribuição para o efeito agregado } \\
\text { dos direcionadores. }\end{array}$ & $\begin{array}{l}\text { Pettan } \\
\text { (2005) }\end{array}$ \\
\hline $\begin{array}{l}\text { Na fase qualitativa buscou-se identificar os indicadores de desempenho por meio } \\
\text { de um fórum com técnicos. A fase quantitativa foi dividida em duas etapas: a } \\
\text { primeira, para identificar a percepção de importância dos indicadores pelos } \\
\text { técnicos; e a segunda, a correlação dos indicadores de desempenho. }\end{array}$ & $\begin{array}{l}\text { Adam, } \\
\text { Oliveira e } \\
\text { Schmidt } \\
(2008)\end{array}$ \\
\hline $\begin{array}{l}\text { Os autores realizaram uma pesquisa bibliográfica para identificar os indicadores de } \\
\text { desempenho. A finalidade de se identificar esses indicadores é disponibilizar um } \\
\text { instrumento que permita avaliação da existência e intensidade de relacionamento } \\
\text { em redes de empresas. }\end{array}$ & $\begin{array}{l}\text { Silveira et al. } \\
\quad(2008)\end{array}$ \\
\hline $\begin{array}{l}\text { Os autores propõem medidas de benchmarking para avaliar como transferir } \\
\text { conhecimento em redes de inovação. O benchmarking pretende ultrapassar a } \\
\text { simples recolha de dados, procurando identificar as melhores práticas, para depois } \\
\text { avaliar e propor ações de melhoria. }\end{array}$ & $\begin{array}{l}\text { Silva et al. } \\
\quad(2008)\end{array}$ \\
\hline $\begin{array}{l}\text { O autor propõe indicadores de desempenho econômico-financeiro por meio da } \\
\text { revisão da literatura para avaliar o impacto das alianças estratégicas no valor de } \\
\text { mercado das empresas. }\end{array}$ & $\begin{array}{l}\text { Klotzle } \\
(2003)\end{array}$ \\
\hline $\begin{array}{l}\text { Para apresentação e análise dos dados, adota-se a seguinte estrutura: a) } \\
\text { performance econômica geral; b) ambiente geral de negócios; c) análise interna do } \\
\text { aglomerado; e d) identificação das questões estratégicas: qualidade e } \\
\text { produtividade no aglomerado. }\end{array}$ & $\begin{array}{l}\text { Mazzaro et } \\
\text { al. (2009) }\end{array}$ \\
\hline $\begin{array}{l}\text { A Visão Baseada em Recurso apresenta um arcabouço teórico que ajuda a } \\
\text { compreender os recursos e como a empresa os utiliza na geração da vantagem } \\
\text { competitiva. Na sequência busca-se analisar os condicionantes e os benefícios } \\
\text { alcançados via configuração interorganizacional. A última etapa do modelo } \\
\text { apresenta as condições e características que uma empresa deve ter para mostrar- } \\
\text { se propensa a inovação. }\end{array}$ & $\begin{array}{l}\text { Martins et al. } \\
\quad(2009)\end{array}$ \\
\hline
\end{tabular}

Fonte: Elaborado pelos autores

Adicionalmente, constata-se que as ferramentas propostas nos periódicos internacionais apresentam maior amadurecimento, o que pode ser explicado pelo maior tempo de existência das redes de empresas nos países dos autores dos artigos.

O Quadro 5 apresenta o cotejamento das ferramentas propostas de AD em redes de PMEs à luz da filiação teórica de $A D$ adotada pelos autores do presente artigo. De forma resumida, os autores filiam-se ao conceito de que as ferramentas de $A D$ devem ter processo para gerar conhecimento no decisor por meio de 
processo para identificar os critérios, mensurar de forma ordinal e cardinal, integrar os critérios e ter processo para gerar ações de aperfeiçoamento. Dessa forma, no Quadro 5 apresentam-se, na primeira coluna, os autores das ferramentas propostas de $A D$ em redes de PMEs, e, nas demais colunas, procurou-se classificar cada ferramenta nos critérios: identifica, mensura, integra e gerencia. Para fazer a classificação, foi analisada cada ferramenta e, em cada critério, utilizou-se Sim, quando a ferramenta desenvolve a etapa; Não, para a etapa ausente na ferramenta; e Parcial, quando o processo proposto pelos autores atende de forma incompleta à etapa.

Quadro 5 - Cotejamento das ferramentas à luz da filiação teórica de Avaliação de Desempenho

\begin{tabular}{|c|c|c|c|c|}
\hline Autores das Ferramentas & Identifica & Mensura & Integra & Gerencia \\
\hline Antonelli e Caroleo (2011) & Não & Não & Não & Não \\
\hline Bititci et al. (2004) & Não & Parcial & Não & Parcial \\
\hline Bititci et al. (2007) & Não & Parcial & Não & Parcial \\
\hline Burlat, Besombes e Deslandres (2003) & Não & Parcial & Não & Parcial \\
\hline Camarinha-Matos e Abreu (2007) & Não & Parcial & Não & Parcial \\
\hline Carpinetti, Galdámez e Gerolamo (2008) & Sim & Parcial & Não & Parcial \\
\hline Carpinetti, Gerolamo e Galdámez (2007) & Sim & Parcial & Não & Parcial \\
\hline Carpinetti e Oiko (2008) & Não & Parcial & Não & Parcial \\
\hline Gerolamo et al. (2008) & Sim & Parcial & Não & Parcial \\
\hline Grando e Belvedere (2006) & Não & Parcial & Não & Parcial \\
\hline Hoffmann e Schlosser (2001) & Não & Parcial & Não & Não \\
\hline Jahn (2009) & Sim & Sim & Sim & Parcial \\
\hline Karaev, Koh e Szamosi (2007) & Não & Parcial & Não & Não \\
\hline Klint e Sjoberg (2003) & Não & Parcial & Não & Não \\
\hline Lima, Guerrini, Carpinetti (2011) & Não & Parcial & Não & Parcial \\
\hline Paniccia (1999) & Não & Parcial & Não & Não \\
\hline Parrilli (2009) & Não & Parcial & Não & Não \\
\hline Rosenfeld (1996) & Não & Parcial & Não & Não \\
\hline Villa (2007) & Sim & Parcial & Não & Parcial \\
\hline Galdamez, Carpinetti e Gerolamo (2009) & Sim & Parcial & Não & Parcial \\
\hline Hansen e Oliveira (2009) & Não & Parcial & Não & Parcial \\
\hline Wegner e Misocsky (2010) & Não & Parcial & Não & Parcial \\
\hline Oliveira e Gomes (2004) & Não & Parcial & Sim & Não \\
\hline Pettan (2005) & Não & Parcial & Não & Não \\
\hline Adam, Oliveira e Schmidt (2008) & Não & Parcial & Não & Não \\
\hline Silveira et al. (2008) & Não & Parcial & Não & Não \\
\hline Silva et al. (2008) & Não & Parcial & Não & Não \\
\hline Klotzle (2003) & Não & Parcial & Não & Não \\
\hline Mazzaro et al. (2009) & Não & Parcial & Não & Não \\
\hline Martins et al. (2009) & Não & Parcial & Não & Não \\
\hline
\end{tabular}

Fonte: Elaborado pelos autores

Contata-se que a maioria das ferramentas apresenta lacunas quando analisadas à luz da filiação teórica de AD adotada pelos autores, principalmente nos 
aspectos identificação dos critérios e integração. Em relação à mensuração, a maioria das ferramentas atende de forma parcial, pois realiza a mensuração de forma ordinal, sendo que pela filiação teórica a mensuração deve ser realizada ordinal e cardinalmente. O trabalho que mais se aproxima da visão dos autores do presente trabalho é a ferramenta proposta por Jahn (2009), que apresenta processo para identificar os critérios, mensura de forma ordinal e cardinal, realiza a integração dos critérios e apresenta um processo parcial de gerenciamento.

A comparação das ferramentas propostas nos artigos publicados em periódicos internacionais versus nacionais permite concluir também que as ferramentas dos periódicos internacionais apresentam maior amadurecimento, pois as ferramentas atendem melhor às etapas da filiação teórica de AD.

\section{CONSIDERAÇÕES FINAIS}

A pesquisa teve por objetivo mapear a importância e as ferramentas de Avaliação de Desempenho (AD) de redes de Pequenas e Médias Empresas (PMEs) publicadas em periódicos nacionais e internacionais. O objetivo geral foi alcançado por meio dos objetivos específicos. O primeiro objetivo específico foi extrair dos artigos publicados em periódicos nacionais e internacionais a visão dos diferentes autores sobre a importância da AD em redes de PMEs e está consolidada nos Quadros 1 e 2, sendo que, de forma geral, a maior importância é o apoio à gestão dessas organizações e, com isso, o aumento de confiança pela transparência das informações.

O segundo objetivo específico foi mapear as ferramentas de AD em redes de PMEs publicados nos periódicos nacionais e internacionais. Esse objetivo foi alcançado pela consolidação e descrição das ferramentas de AD em redes de PMEs que é apresentada nos Quadros 3 e 4. Conclui-se que as principais dimensões de análise usadas pelas ferramentas são: intercâmbio de informações; gerenciamento de valor em redes; nível de maturidade das redes; consideração das empresas individuais e a rede como um todo; os benefícios da colaboração; capital social; eficiência coletiva; especialização; inovação; confiança e ciclo de vida da rede; 
existência e intensidade do relacionamento entre os parceiros e comparação de desempenho entre os parceiros.

O terceiro objetivo específico foi cotejar a literatura nacional versus internacional em relação à importância e as ferramentas de $A D$ em redes PMEs. Esse objetivo foi alcançado por meio dos argumentos apresentados na análise da importância e das ferramentas de $A D$ em redes de PMEs, sendo que, em relação à importância da AD em redes de PMEs, conclui-se que os apresentados na literatura nacional e internacional são similares e complementares, ou seja, não existe grandes divergências sobre a importância da $A D$ em redes de PMEs, e, em relação às ferramentas, constata-se que a literatura internacional apresenta maior amadurecimento em termos de pesquisa sobre o tema.

O quarto objetivo específico foi analisar criticamente as ferramentas de AD em redes de PMEs em função da filiação teórica adotada no presente estudo. Esse objetivo foi alcançado à medida que se fez o cotejamento de todas as ferramentas identificadas no estudo em relação à filiação teórica de AD (Quadro 5). Conclui-se que as ferramentas propostas na literatura apresentam lacunas/oportunidades de pesquisa, quando analisadas à luz da filiação teórica. A principal lacuna diz respeito à identificação e integração dos critérios. No entanto, a maioria das ferramentas apresenta deficiências em relação à mensuração e ao gerenciamento.

O presente estudo corrobora a literatura ao concluir, da mesma forma, que as tentativas de oferecer um quadro consistente de AD para redes ainda são poucos (WEGNER e MISOCSKY, 2010), e que o tema Redes de PMEs apresenta, em termos de pesquisa, diversas lacunas a serem preenchidas (NEVES et al., 2011), sendo que uma lacuna importante é a consolidação de ferramentas de AD em redes de PMEs (GALDÁMEZ, CAPINETTI e GEROLAMO, 2009), principalmente pela busca de adaptação de modelos desenvolvidos para empresas individuais e sua aplicação em redes de cooperação (LIMA, GUERRINI e CARPINETTI, 2011). Além disso, o presente artigo conclui que as ferramentas propostas na literatura nacional e internacional apresentam lacunas na identificação dos objetivos/critérios, na mensuração dos critérios, na integração dos critérios e no processo de gerenciamento do desempenho. 
No entanto, os autores deste artigo também corroboram a literatura ao observar a importância das ferramentas de AD para a gestão de redes de empresas (PANICCIA, 1999; WEGNER e MISOCSKY, 2010; ANTONELLI e CAROLEO, 2011). Porém, essas ferramentas devem, à luz da filiação teórica do artigo, apresentar processo para gerar conhecimento nos decisores por meio da identificação, mensuração, integração dos critérios e gerenciamento do desempenho.

A pesquisa apresenta as seguintes limitações: (i) a busca restringiu-se aos artigos disponibilizados nos periódicos do portal da CAPES; (ii) o processo de busca se apresenta em várias etapas subjetivas; e, (iii) a busca não abrangeu artigos publicados em congressos, livros, teses e dissertações. Para futuras pesquisas, sugere-se ampliar a busca de artigos em congresso, livros, teses e dissertações e desenvolver uma ferramenta de $A D$ para redes de PMEs que atenda aos aspectos relacionados na filiação teórica de AD adotada pelos autores.

\section{REFERÊNCIAS}

ADAM, C. R.; OLIVEIRA, J. H. R.; SCHMIDT, S. Proposição de indicadores para avaliação de desempenho de redes de cooperação gaúchas. Redes: Revista do Desenvolvimento Regional, Santa Cruz do Sul, v. 13, n. 3, p. 218-240, 2008.

ANTONELLI, D.; CAROLEO, B. An integrated methodology for the analysis of collaboration in industry networks. Journal of Intelligent Manufacturing, v. 23, n. 6, p. 2443-2450, 2012. http://dx.doi.org/10.1007/s10845-011-0510-z

BARDIN, L. Análise de Conteúdo. Lisboa: edições 70, 2004.

BITITCI, U. S.; MARTINEZ, V.; ALBORES, P.; PARUNG, J. Creating and managing value in collaborative networks. International Journal of Physical Distribution \& Logistics

Management, v. 34, n. 3, p. 251-268, 2004. http://dx.doi.org/10.1108/09600030410533574

BITITCI, U. S.; TURNER, T.; MACKAY, D.; KEARNEY, D.; PARUNG, J. WALTERS, D. Managing synergy in collaborative enterprises. Production Planning \& Control, v. 18, n. 6 , p. 454-465, 2007. http://dx.doi.org/10.1080/09537280701494990

BORTOLUZZI, S. C; ENSSLIN, S. R; ENSSLIN, L; VICENTE, E. F. R. Práticas de avaliação de desempenho organizacional em pequenas e médias empresas: investigação em uma empresa de porte médio do ramo moveleiro. Revista Produção online, v. 10, n. 3, p. 551576, 2010. http://dx.doi.org/10.14488/1676-1901.v10i3.244 
BORTOLUZZI, S. C; ENSSLIN, S. R; ENSSLIN, L. Modelo multicritério para apoiar decisões relacionadas ao desempenho da área de mercado de uma empresa de informática. Revista Produção online, v. 13, n. 1, p. 2-36, 2013. http://dx.doi.org/10.14488/1676-1901.v13i1.795

BURLAT, P.; BESOMBES, B.; DESLANDRES, V. Constructing a typology for networks of firms. Production Planning \& Control, v. 14, n. 5, p. 399-409, 2003.

CAMARINHA-MATOS, L. M.; ABREU, A. Performance indicators for collaborative networks based on collaboration benefits. Production Planning \& Control, v. 18, n. 7, p. 592-609, 2007. http://dx.doi.org/10.1007/0-387-29360-4 28

CARPINETTI, L. C. R.; GALDÁMEZ, E. V. C.; GEROLAMO, M. C. A measurement system for managing performance of industrial clusters: A conceptual model and research cases. International Journal of Productivity and Performance Management, v. 57, n. 5, p. 405419, 2008. http://dx.doi.org/10.1108/17410400810881854

CARPINETTI, L. C. R.; GEROLAMO, M. C.; GALDÁMEZ, E. V. C. Continuous Innovation and Performance Management of SME Clusters. Creativity and Innovation Management, v. 16, n. 4, p. 376-385, 2007. http://dx.doi.org/10.1111/j.1467-8691.2007.00448.x

CARPINETTI, L. C. R.; OIKO, O. T. Development and application of a benchmarking information system in clusters of SMEs. Benchmarking: An International Journal, v. 15, n. 3, p. 292 306, 2008. http://dx.doi.org/10.1108/14635770810876601

CASSANEGO JR, P; MAEHLER, A. E. O programa de apoio aos arranjos produtivos locais (APL`S) no RS: Uma política de criação de vantagem competitiva as empresas de micro e pequeno porte. RDE Revista de Desenvolvimento Econômico, v. 12, n. 21, p. 62-73, 2010.

CASTRO, C. M. A prática da pesquisa. São Paulo: McGraw-Hill do Brasil, 1977.

DUTRA, A.; RIPOLL-FELIU, V.M.; FILLOL, A. G.; ENSSLIN, S. R.; ENSSLIN, L. The construction of knowledge from the scientific literature about the theme seaport performance evaluation. International Journal of Productivity and Performance Management, v. 64, iss 2, p. $243-269,2015$. http://dx.doi.org/10.1108/IJPPM-01-2014-0015

ENSSLIN, S. R.; ENSSLIN, L.; IMLAU, J. M.; CHAVES, L. C. Processo de mapeamento das publicações científicas de um tema: portfólio bibliográfico e análise bibliométrica sobre avaliação de desempenho de cooperativas de produção agropecuária. Revista de Economia e Sociologia Rural, v. 52, p. 587-608, 2014.

http://dx.doi.org/10.1590/S0103-20032014000300010

ENSSLIN, L.; GIFFHORN, E.; ENSSLIN, S. R.; PETRI, S. M.; VIANNA, W. B. Avaliação do Desempenho de Empresas Terceirizadas com o Uso da Metodologia Multicritério de Apoio à Decisão- Construtivista. Revista Pesquisa Operacional, v.30, n. 1, p. 125-152, 2010. http://dx.doi.org/10.1590/S0101-74382010000100007 
GALDÁMEZ, E. V. C.; CARPINETTI, L. C. R.; GEROLAMO, M. C. Proposta de um sistema de avaliação do desempenho para arranjos produtivos locais. Gestão \& Produção, v. 16, n. 1, p. 133-151, 2009. http://dx.doi.org/10.1590/S0104-530X2009000100013

GEROLAMO, M. C.; CARPINETTI, L. C. R.; SELIGER, G.; GALDÁMEZ, E. V. C. Performance management of regional clusters and SME cooperation networks. International Journal of Business Excellence, v. 1, n. 4, p. 457-483, 2008. http://dx.doi.org/10.1504//JBEX.2008.018844

GIL, A. C. Como elaborar projetos de pesquisa. 3. ed. São Paulo: Atlas, 1999.

GRANDO, A.; BELVEDERE, V. District's manufacturing performances: A comparison among large, small-to-medium-sized and district enterprises. International Journal of Production Economics, v. 104, n. 1, p. 85-99, 2006. http://dx.doi.org/10.1016/j.ijpe.2005.01.007

HANSEN, P. B.; OLIVEIRA, L. R. Proposta de modelo para avaliação sistêmica do desempenho competitivo de arranjos produtivos: o caso do arranjo coureiro calçadista do Vale dos Sinos (RS - Brasil). Revista Produto \& Produção, v. 10, n. 3, p. 61-75, 2009.

HOFFMANN, W. H.; SCHLOSSER, R. Success factors of strategic alliances in small and medium-sized enterprises an empirical survey. Long Range Planning, v. 34, n. 3, p. 357381, 2001. http://dx.doi.org/10.1016/S0024-6301(01)00041-3

JÄHN, H. Value-added process-related performance analysis of enterprises acting in cooperative production structures. Production Planning \& Control, v. 20, n. 2, p. 178-190, 2009. http://dx.doi.org/10.1080/09537280802705088

KARAEV, A.; KOH, S. C. L.; SZAMOSI, L. T. The cluster approach and SME competitiveness: A review. Journal of Manufacturing Technology Management, v. 18, n. 7, p. 818-835, 2007. http://dx.doi.org/10.1108/17410380710817273

KLINT, M. B.; SJOBERG, U. Towards a comprehensive SCP-model for analysing strategic networks/alliances. International Journal of Physical Distribution \& Logistics

Management, v. 33, n. 5, p. 408-426, 2003. http://dx.doi.org/10.1108/09600030310481988

KLOTZLE, M. C. O impacto da formação de alianças estratégicas no valor de mercado e no desempenho econômico-financeiro das empresas. Caderno de Pesquisa em

Administração, v. 10, n. 4, p. 33-46, 2003.

LACERDA, R. T. O., ENSSLIN, L.; ENSSLIN, S. R. Uma Análise Bibliométrica da Literatura Sobre Estratégia e Avaliação De Desempenho. Gestão \& Produção, v.19, n.1. 2012. http://dx.doi.org/10.1590/S0104-530X2012000100005

LIMA, P. R. H.; GUERRINI, F. M.; CARPINETTI, L. C. R. Performance measurement in collaborative networks: a proposal of performance indicators for the manufacturing industry. International Journal of Business Excellence, v. 4, n. 1, p. 61-79, 2011.

http://dx.doi.org/10.1504//JBEX.2011.037249

MARTINS, D. A.; SANTOS, E. M.; CAMPUS, A. J. M.; FEITOR, C. D. C.; GUERRA, L. C. C. B.; FORBELONI, J. V. Arranjos Produtivos Locais: contribuições para uma análise 
longitudinal e complexa dos aspectos estratégicos. Aríus-Revista de Ciências Humanas e Artes, v. 15, n. 2, p. 73-85, 2009.

MAZZARO, L. G.; OLIVA, F. L.; GRISI, C. C. H.; DROUVOT, H.; CRISPIM, S.; GASPAR, M. A. A competitividade nos Clusters da indústria de borracha do sudeste asiático. Revista de Ciências da Administração, v. 11, n. 23, p. 65-86, 2009.

http://dx.doi.org/10.5007/2175-8077.2009v11n23p65

NEUMANN, L.; HEXSEL, A.; BALESTRIN, A. Desafios à cooperação em aglomerados produtivos: um estudo de caso no segmento de malhas do sul do Brasil. BASE - Revista de Administração e Contabilidade da Unisinos, v. 8, n. 3, p. 220-230, 2011.

NEVES, M. P. S.; DIEHL, C. A.; HANSEN, P. B.; BECKER, G. V. Análise do processo de coopetição em redes horizontais de pequenas e médias empresas do Rio Grande do Sul. BASE - Revista de Administração e Contabilidade da Unisinos, v. 8, n. 3, p. 243-260, 2011.

OLIVEIRA, H. C.; GOMES, A. P. Eficiência nas Empresas de Diagnóstico Humano do Cluster Mineiro de Biotecnologia. Contextus Revista Contemporânea de Economia e Gestão, v. 2, n. 2, p. 37-47, 2004.

PANICCIA, I. The performance of IDs. Some insights from the Italian case. Human Systems Management, v. 18, n. 2, p. 141-159, 1999.

PARRILLI, M. D. Collective efficiency, policy inducement and social embeddedness: Drivers for the development of industrial districts. Entrepreneurship \& Regional Development, v. 21, n. 1, p. 1-24, 2009. http://dx.doi.org/10.1080/08985620801886513

PEREIRA, B. A. D.; VENTURINI, J. C. Identificação dos fatores determinantes do desempenho das empresas inseridas em redes horizontais. Revista de Ciências da Administração, v. 8, n. 16, p. 260-279, 2006.

PETTAN, K. B. Análise comparativa do desempenho da competitividade das agroindústrias familiares no oeste de Santa Catarina em relação ao ambiente institucional. Cadernos de Ciência \& Tecnologia, v. 22, n. 3, p. 667-689, 2005.

RICHARDSON, R. J. Pesquisa Social: Métodos e Técnicas. 3. ed. São Paulo: Atlas, 2008.

ROSA, F. S.; ENSSLIN, S. R.; ENSSLIN, L.; LUNKES. Evidenciação ambiental para gestão interna: um estudo sobre as potencialidades e oportunidades do tema. Engenharia

Sanitária e Ambiental, v. 16, p. 157-166, 2011. http://dx.doi.org/10.1590/S1413-41522011000200009

ROSENFELD, S. A. Does cooperation enhance competitiveness? Assessing the impacts of inter-firm collaboration. Research Policy, v. 25, edição especial, p. 247-263, 1996. http://dx.doi.org/10.1016/0048-7333(95)00835-7

SILVA, M. J.; LEITAO, J.; LEITÃO, D.; RAPOSO, M. Como transferir conhecimento em redes de inovação? Uma proposta de «benchmarking». Revista Portuguesa e Brasileira de Gestão, v. 7, n. 2, p. 22-35, 2008. 
SILVEIRA, M. A. P.; LANÇA, E. C. R.; PESTANA, A. L.; SANTOS, C. P. Indicadores para o uso de sistemas interorganizacionais e relacionamento em rede entre empresas. RESI-

Revista Eletrônica de Sistemas de Informação, v. 7, n.2, 2008.

VILLA, A. Analysing industrial district performances: A structured approach. Annual Reviews in Control, v. 31, n. 1, p. 107-118, 2007.

http://dx.doi.org/10.1016/j.arcontrol.2007.03.002

WEGNER, D.; MISOCSKY, M. C. avaliação de desempenho de redes de pequenas empresas: contribuições da abordagem da produção de sentido. Revista O\&S, v. 17, n. 53, p. 456-361, 2010. http://dx.doi.org/10.1590/S1984-92302010000200007

\section{(a) (1)}

Artigo recebido em 10/10/2013 e aceito para publicação em 16/09/2015 DOI: http://dx.doi.org/ 10.14488/1676-1901.v15i4.1689 